\title{
Analysis of the mechanism of quinolone resistance in nalidixic acid-resistant clinical isolates of Salmonella serotype Typhimurium
}

\author{
J. RUIZ, D. CASTRO*, P. GOÑI, J. A. SANTAMARIA*, J. J. BORREGO* and J. VILA \\ Department of Microbiology, Hospital Clinic, School of Medicine, University of Barcelona, Villarroel 170,08036 \\ Barcelona and *Department of Microbiology, School of Sciences, University of Málaga, 29071 Málaga, Spain
}

\begin{abstract}
Over a period of 2.5 years, 42 cases of gastro-enteritis caused by nalidixic acid-resistant Salmonella serotype Typhimurium occurred in Malaga. The epidemiological relationship among the strains involved was investigated by analysis of plasmid profile and of chromosomal DNA by pulsed-field gel electrophoresis (PFGE). Despite having different plasmid profiles, all 42 nalidixic-acid resistant Typhimurium isolates had evolved from one clone as shown by analysis of chromosomal DNA by PFGE. The mechanism of quinolone resistance in these Typhimurium isolates was also investigated. Analysis of outer-membrane proteins and lipopolysaccharide from quinolone-susceptible and -resistant clinical isolates tested showed no differences. All nalidixic acid-resistant isolates had MICs for ciprofloxacin of $0.25 \mathrm{mg} / \mathrm{L}$ and for nalidixic acid of $1024 \mathrm{mg} / \mathrm{L}$. Polymerase chain reaction fragments of $285 \mathrm{bp}$, containing the quinolone resistancedetermining region of the gyrA gene, and of $237 \mathrm{bp}$, containing the region of parC homologous to the quinolone resistance-determining region of the $\operatorname{gyr} \mathrm{A}$ gene, were sequenced. All resistant isolates presented a change at Ser-83 to Phe in the GyrA protein, but no changes were observed in the ParC protein. These findings indicated that this mutation in gyra plays a major role in the acquisition of nalidixic-acid resistance in clinical isolates of Typhimurium.
\end{abstract}

\section{Introduction}

Acute salmonellosis in healthy people usually takes the form of a self-limited enterocolitis not requiring specific therapy. However, in some cases antibiotic therapy may be necessary. Despite susceptibility to various antimicrobial agents in vitro, Salmonella serotypes causing systemic infection have been documented to respond to only a few antibiotics. The most likely explanation for this lack of correlation between in-vitro and in-vivo efficacy appears to be the need to achieve adequate intracellular (macrophage) concentrations of antimicrobial agents. Thus, the fluoroquinolones, with their demonstrated in-vitro activity against Salmonella strains and their ability to penetrate cells, should be effective agents against these pathogens.

Ciprofloxacin has been used successfully in the treatment of chronic salmonella carriers. In acute

Received 14 Aug. 1996; revised version accepted 11 Dec. 1996.

Corresponding author: Dr J. Vila. infections the drug significantly shortens the duration of symptoms and the faecal excretion of salmonellae [1-3]. Moreover, the development of resistance during therapy of enterocolitis caused by Salmonella serotypes has been reported [4]. Quinolone resistance that developed during ciprofloxacin therapy of two patients with serotype Typhimurium infections has been described [5]. Analysis of sequential isolates suggested that resistance was due to a mutation in gyr A in one patient and to reduced quinolone accumulation through an unknown mechanism in the other. In a clinical isolate of Typhimurium with a high level of fluoroquinolone resistance (MIC of ciprofloxacin, $32 \mathrm{mg} / \mathrm{L}$ ), it was shown that alterations of both gyrA and $g y r \mathrm{~B}$ genes were responsible for the high level of resistance [6]. A study of spontaneous quinolone-resistant mutants of Typhimurium [7] found mutations in both amino acids equivalent to Ser-83 and Asp-87 in Escherichia coli. The sequence of the quinolone resistancedetermining region of gyrA of clinical isolates of Typhimurium has been reported [8]. The parC gene has also been implicated in quinolone resistance in E. coli [9, 10]. 
This study characterised nalidixic acid-resistant clinical isolates of Typhimurium and determined the molecular basis for quinolone resistance by means of DNA sequencing of the quinolone resistance-determining regions of gyrA and of parC genes, quinolone uptake and determination of lipopolysaccharide (LPS) and outer-membrane proteins (OMPs).

\section{Materials and methods}

\section{Bacterial strains}

Forty-two nalidixic acid-resistant isolates of Typhimurium were obtained from stool samples in 1989-1992. Two quinolone-susceptible strains were used as controls in the determination of the mechanism of quinolone resistance.

\section{Plasmid analysis}

Plasmid DNA was extracted from lysed cells by the technique of Kado and Liu [11].

\section{Chromosomal DNA analysis by pulsed-field gel electrophoresis}

Genomic DNA was prepared in agarose plugs and treated as described previously [12]. One DNA insert was incubated overnight with $50 \mathrm{U}$ of Xho I (Promega Co., Madison, WI, USA) according to the manufacturer's instructions. Resultant DNA fragments were separated in an agarose $1 \% \mathrm{w} / \mathrm{v}$ gel (BioRad, Richmond, CA, USA) that was prepared and run in Tris-borate-EDTA buffer in a contour-clamped homogeneous field apparatus (CHEF-DR2, BioRad). The conditions for electrophoresis were $200 \mathrm{~V}$ for $19 \mathrm{~h}$ with pulse times of 5-8 s. Thereafter, gels were stained with ethidium bromide and photographed.

\section{Antimicrobial susceptibility testing}

Susceptibility testing was performed by an agardilution method and in accordance with the guidelines of the National Committee for Clinical Laboratory Standards [13]. About $10^{4} \mathrm{cfu}$ of each isolate were inoculated by means of a multipoint replicator on to freshly prepared medium containing serial dilutions of ciprofloxacin (Bayer, Leverkusen, Germany) or nalidixic acid (Prodesfarma, Barcelona, Spain).

Antimicrobial susceptibility tests were also performed by an agar-diffusion disk method as advocated by the National Committee for Clinical Laboratory Standards [14]. Mueller-Hinton agar and antimicrobial disks (ampicillin, $10 \mu \mathrm{g}$; gentamicin, $10 \mu \mathrm{g}$; tetracycline, $30 \mu \mathrm{g}$; chloramphenicol, $30 \mu \mathrm{g}$ and cephalothin, $30 \mu \mathrm{g}$ ) were obtained from BBL Microbiology Systems, Becton Dickinson, Cockeysville, MD, USA.

\section{Ciprofloxacin uptake}

The uptake of ciprofloxacin was determined by the method of Chapman and Georgopapadokou with the modifications introduced by Mortimer and Piddock [15].

\section{Preparation of OMPs and LPS}

OMP analysis was performed by SDS-PAGE as described elsewhere [16]. Cytoplasmic membranes were selectively solubilised with sodium lauryl sarcosinate [17]. Proteins were detected in gels by staining with Coomassie Brillant Blue.

The extraction of LPS followed the method of Hitchcock and Brown [18]. Samples were electrophoresed in discontinuous polyacrylamide-SDS gels 4.5$15 \% \mathrm{w} / \mathrm{v}$. LPS was detected by silver staining [19].

Amplification and DNA sequencing of quinolone resistance-determining region in gyrA and parC genes

PCR was performed as described elsewhere [20]. The PCR reaction was performed in a DNA Thermal Cycler 480 (Perkin-Elmer Cetus, Emeryville, CA, USA). Primers and free nucleotides were removed with a QiaQuick spin PCR purification kit (Qiagen, Inc., Chatsworth, CA, USA) according to the manufacturer's instructions; the sample was directly processed for DNA sequencing with TaqDyeDeoxyTerminator Cycle Sequencing kit (Applied Biosystems) and analysed in an automatic DNA sequencer (Applied Biosystems 373A). When the PCR product was digested with Hinfl, $20 \mu \mathrm{l}$ of PCR mixture were incubated for $2.5 \mathrm{~h}$ with $10 \mathrm{U}$ of restriction endonuclease as recommended by the manufacturer; digestion products were separated by electrophoresis in NuSieve $1.5 \% \mathrm{w} / \mathrm{v}$ and agarose $1 \% \mathrm{w} / \mathrm{v}$ gels.

The EMBL accession number for the partial sequence of the quinolone-susceptible strain SM-4 and the quinolone-resistant strain S38 of Typhimurium gyr A gene is X86695.

\section{Results}

The epidemiological relationship among 42 nalidixic acid-resistant isolates of Typhimurium obtained from stool samples in the period 1989-1992 was examined by plasmid analysis and chromosomal DNA analysis by PFGE. Plasmid analysis revealed five profiles (Table 1). All 42 nalidixic acid-resistant clinical isolates of Typhimurium contained 1-4 plasmids. A predominant plasmid with a mol. wt of $3.7 \mathrm{~kb}$ was found in four of the five profiles. Antimicrobial susceptibility was not used as an epidemiological marker because all strains tested showed the same antibiogram, i.e., were 
Table 1. Epidemiological markers for discrimination of nalidixic acid-resistant clinical isolates of Typhimurium

\begin{tabular}{lcc}
\hline Group no. & Plasmid profile $(\mathrm{kb})$ & PFGE type \\
\hline I & $1.8,2.3,23.2,57.5$ & $\mathrm{~A}_{1}$ \\
II & $2.0,2.3,3.7$ & $\mathrm{~A}_{2}$ \\
III & $2.6,3.7$ & $\mathrm{~A}_{2}$ \\
IV & $3.1,3.7,4.1,57.5$ & $\mathrm{~A}_{2}$ \\
V & 3.7 & $\mathrm{~A}_{2}$ \\
\hline
\end{tabular}

susceptible to all of the antibiotics except nalidixic acid. Analysis of chromosomal DNA restriction patterns by PFGE revealed two patterns (Fig. 1) which, however, were considered to be subtypes, different in only two fragments. The percentage similarity among the two different subtypes of restriction fragment, determined by the unweighted pair group method using averages (UPGMA) algorithm, was $>86 \%$.

To determine whether decrease in permeability to fluoroquinolones contributed to resistance, the accumulation of ciprofloxacin, the integrity of LPS and the composition of their OMPs were analysed. The uptake of ciprofloxacin is shown in Table 2. Kinetic studies showed that accumulation was complete within $5 \mathrm{~min}$; thus, that time point was used to calculate the accumulation level. No significant differences were noted on the ciprofloxacin uptake among the strains analysed. Although slight differences were observed in OMP profiles, there was no clear relationship between decreased levels of expression of specific OMPs and quinolone accumulation (Fig. 2a). The long chain Opolysaccharide component of LPS was visualised in LPS preparations of both nalidixic-susceptible and resistant clinical isolates (Fig. 2b).

To determine whether resistance was due to altered DNA gyrase or topoisomerase IV, or both, the quinolone resistance-determining region of gyrA gene and the region of parC homologous to the quinolone resistance-determining region of the gyr A gene from Typhimurium were amplified and sequenced. A 343-bp gyrA-gene fragment was amplified from each isolate (Fig. $3 \mathrm{~b}$ ) and $285 \mathrm{bp}$ of sequence was determined with
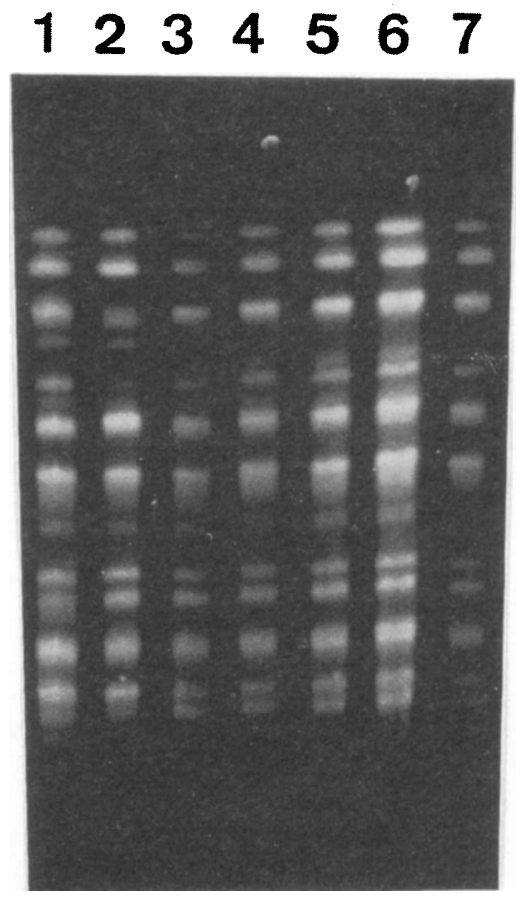

Fig. 1. PFGE of Xho I-digested genomic DNA of isolates of Typhimurium showing: lanes 1 and 2 , subtype $A_{1} ; 3-$ 7, subtype $A_{2}$.

the lower primer (Fig. 3a). The nucleotide sequence exhibited a $91 \%$ similarity to the corresponding region in E. coli [21], whereas the derived amino-acid sequence of Typhimurium GyrA protein showed $100 \%$ identity with that of $E$. coli. The gyrA-gene fragments from quinolone-resistant clinical isolates differed at one nucleotide position compared with those of susceptible isolates. They carried a $\mathrm{C} \rightarrow \mathrm{T}$ transition equivalent to this at position 251 in $E$. coli, resulting in a Ser-83 $\rightarrow$ Phe substitution in the gyrase A protein. Hinfl digestion of the PCR product from a quinolone-susceptible Typhimurium strain generated four fragments of $149,99,52$ and $43 \mathrm{bp}$. One of the Hinfl restriction sites in isolates carrying a mutation at codon 83 was abolished, giving three fragments (151, 149 and $43 \mathrm{bp}$ ) after digestion with Hinfl (Fig. 3b).

Table 2. Quinolone resistance mutations in the gyrA gene of clinical isolates of Typhimurium

\begin{tabular}{|c|c|c|c|c|}
\hline \multirow[b]{2}{*}{ Strain no. } & \multirow[b]{2}{*}{ Ciprofloxacin uptake* } & \multicolumn{2}{|c|}{$\operatorname{MIC}(\mathrm{mg} / \mathrm{L})$} & \multirow[b]{2}{*}{ Amino-acid change ${ }^{\dagger}$} \\
\hline & & Cip & Nal & \\
\hline $\mathrm{S} 18^{\ddagger}$ & $30.53 \mathrm{SD} 4.35$ & $<0.06$ & 4 & - \\
\hline $\mathrm{S} 4^{\ddagger}$ & 27.54 SD 2.67 & $<0.06$ & 4 & - \\
\hline$I^{\S}$ & 30.09 SD 2.57 & 0.25 & 1024 & Ser- 83 to Phe \\
\hline $\mathrm{II}^{\S}$ & 23.14 SD 1.28 & 0.25 & 1024 & Ser- 83 to Phe \\
\hline $\mathrm{III}^{\S}$ & 21.19 SD 3.4 & 0.25 & 1024 & Ser -83 to Phe \\
\hline $\mathrm{IV}^{\S}$ & 22.73 SD 7.74 & 0.25 & 1024 & Ser- 83 to Phe \\
\hline $\mathrm{V}^{\S}$ & 29.29 SD 6.85 & 0.25 & 1024 & Ser- 83 to Phe \\
\hline
\end{tabular}

Cip, ciprofloxacin; Nal, nalidixic acid.

* Results expressed as $\mathrm{mg}$ of ciprofloxacin $/ \mathrm{mg}$ of bacteria, are averages (and SD) of three assays.

${ }^{\dagger}$ Amino-acid position equivalent to Ser-83 of $E$. coli.

${ }^{\ddagger}$ Nalidixic-acid susceptible clinical isolates used as controls.

${ }^{\S}$ Naladixic-acid resistant clinical isolates representative of epidemiological groups indicated by plasmid analysis (see Table 1). 
a

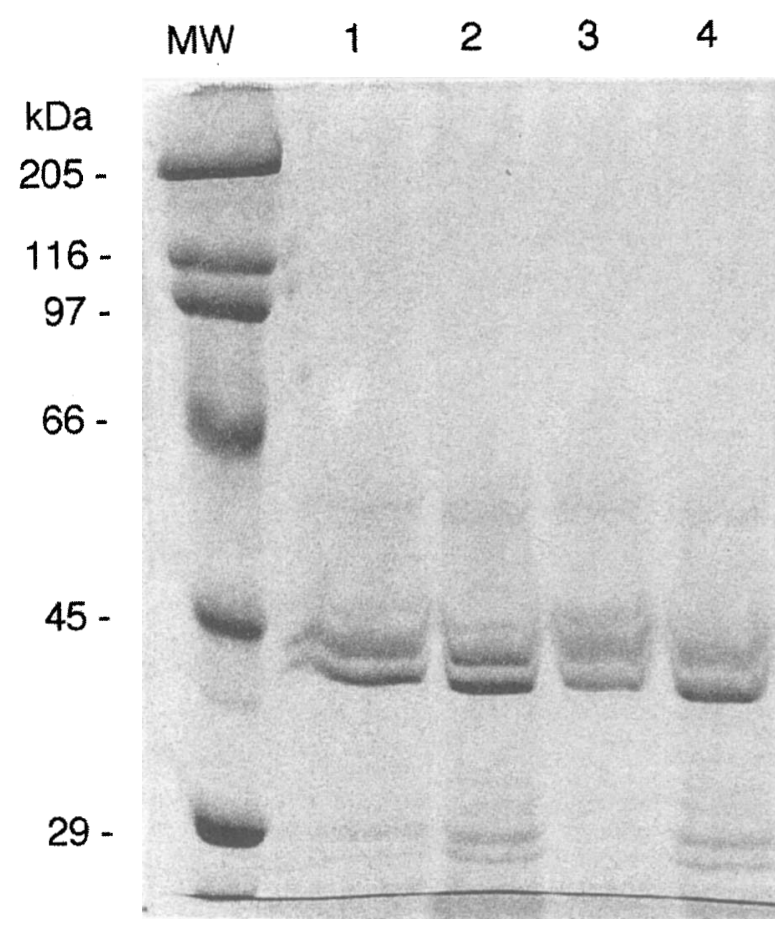

b

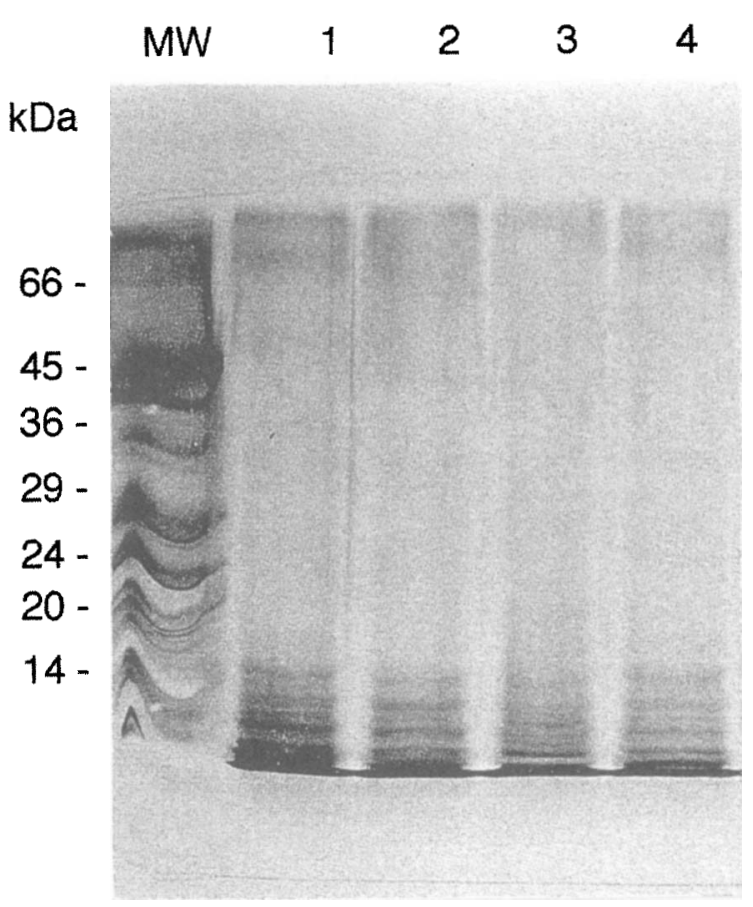

Fig. 2. Profiles of (a) OMP and (b) LPS observed among clinical isolates of Typhimurium. Lanes 1 and 2, quinolone susceptible; $\mathbf{3}$ and 4 , quinolone resistant; MW, molecular mass markers (kDa) are shown.

a

$\begin{array}{llllllllllllllllllll}D & V & I & G & K & Y & H & P & H & G & f & S\end{array}$ GAC GTA ATC GGT AAA TAC CAT CCC CAC GGC GAT TCC $\begin{array}{lllllllllllll}A & V & Y & D & T & I & V & R & M & A & Q & P\end{array}$ GCA GIG TAT GAC ACC ATC GIT CGI ATG GCG CAG CCA $\begin{array}{llllllllllll}F & S & L & R & Y & M & L & V & D & G & Q & G\end{array}$ TTC TCG CIG CGT TAC ATG CIG GIG GAT GGT CAG GGT $\begin{array}{llllllllllllllllll}N & F & G & S & I & D & G & D & S & A & A & A\end{array}$ AAC TIC GGI TCT ATT GAC GGC GAC TCC GCG GCG GCA $\begin{array}{lllllllllllll}M & R & Y & T & E & I & R & L & A & K & I & A\end{array}$ ATG CGT TAT ACG GAG ATC CGI CTG GCG AAA ATC GCC $\begin{array}{llllllllllll}H & E & L & M & A & D & L & E & K & E & T & V\end{array}$ CAC GAA CTG ATG GCC GAT CIC GAA AAA GAG ACG GIG $\begin{array}{llllllllllll}D & F & V & D & N & Y & D & G & T & E & K & I\end{array}$ GAT TTC GIG GAT AAC TAT GAC GGT ACG GAA AAA ATT $\begin{array}{llllllllllllllllll}P & D & V & M & P & T & K & I & P & N & L\end{array}$ CCG GAC GIC ATG CCG ACC AAA ATT CCG AAT CIT b

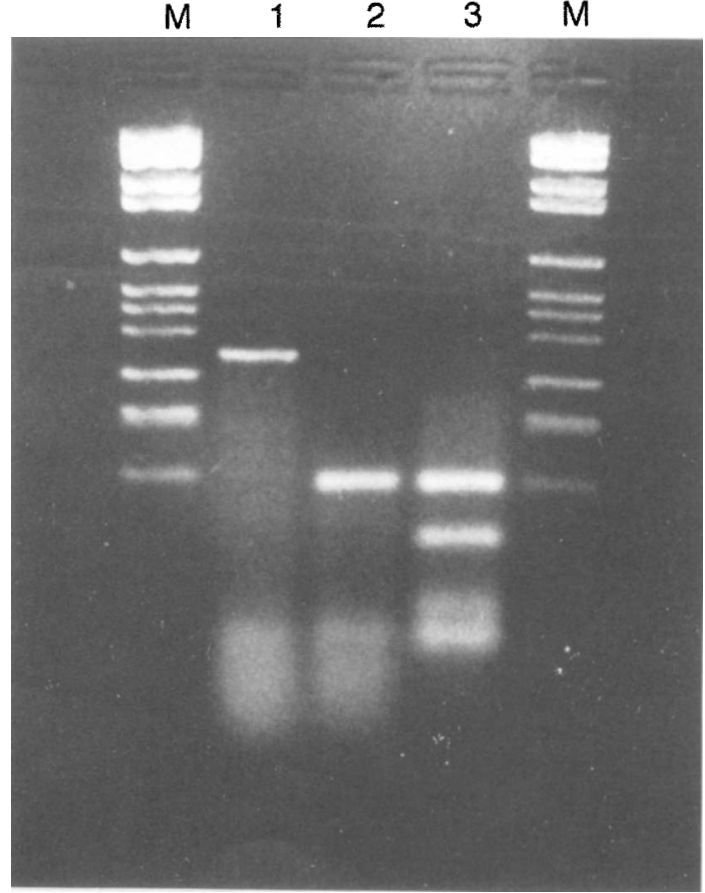

Fig. 3. (a) Nucleotide sequence of quinolone resistance-determining region of the gyrA gene of Typhimurium with deduced amino-acid sequence shown above the nucleotide sequence and positions of restriction sites for Hinfl indicated by arrows. (b) Detection of mutation in gyr A of clinical isolates of Typhimurium by HinfI RFLP analysis of PCR products. Lane 1, non-digested PCR product; 2, HinfI-digested products of quinolone-resistant clinical isolates; 3, HinfI-digested products of quinolone-susceptible clinical isolates; M, mol.-wt markers (VI, Boehringer). 
The mutations in the gyrA gene leading to amino-acid changes are shown in Table 2. All clinical isolates for which the MIC $(\mathrm{mg} / \mathrm{L})$ of ciprofloxacin was $>0.125$ and of nalidixic acid was $>256$ showed a change at Ser-83 to Phe, whereas in two quinolone-susceptible strains changes in this amino acid were not observed. The sequenced regions extended from amino acid 68 to 166 and no other amino acid changes were observed. A 291-bp parC gene fragment was amplified from each isolate and $237 \mathrm{bp}$ of sequence were determined with the upper primer. The sequence showed no mutations with regard to the original sequence described by Luttinger et al. [22].

\section{Discussion}

In the period 1989-1992, 42 nalidixic acid-resistant clinical isolates of Typhimurium were isolated in Malaga, Spain. Plasmid fingerprinting techniques have been applied successfully for typing clinical isolates of Salmonella [23, 24]. However, it is well known that bacteria can gain or lose plasmids and that repeated subculture and storage may also result in plasmid loss. Although plasmid profiles allowed distribution of the strains into five groups, chromosomal DNA analysis by PFGE showed that the clinical isolates analysed were closely related. Thus, this local increase of nalidixic acid-resistant Typhimurium was likely to be due to the spread of a resistant clone. Low-frequency restriction analysis of chromosomal DNA by PFGE is a wellestablished procedure for typing several kinds of bacteria; however, it has been used in Typhimurium only to determine physical mapping of the chromosome and not for epidemiological studies $[25,26]$.

The emergence of multiresistant strains of Salmonella has led to the re-evaluation of fluoroquinolones for treatment of salmonellosis. However, there have been several reports of treatment failure resulting from the emergence of quinolone resistance in Typhimurium in the course of therapy. The mechanisms of quinolone resistance have been well characterised in other members of Enterobacteriaceae but not in Salmonella. Two mechanisms of quinolone resistance have been described: (a), mutations in $g y r \mathrm{~A}, \operatorname{gyr} \mathrm{B}$ or parC genes; and (b), reduced levels of quinolone uptake. With an LPS-deficient mutant of Typhimurium and porin-deficient mutants of E. coli, Hirai et al. [27] showed that quinolones with a low relative hydrophobicity (e.g. nalidixic acid) appeared to permeate through OMP porin $\mathrm{F}$, whereas quinolones with a high relative hydrophobicity appeared to permeate through both OMP porin $\mathrm{F}$ and phospholipid bilayers. In the present study, comparison of the composition of OMPs and LPS from clinical isolates of Typhimurium showed no variation among susceptible and resistant isolates. These results, taken together with data on ciprofloxacin accumulation, show that in these strains permeability does not play a role in the acquisition of quinolone resistance. The results agree with those of Lewin et al. [28] who found no difference in OMP profiles of four nalidixic acid-resistant Typhimurium isolates, whereas Howard et al. [29] found substantially reduced levels of expression of OMP $F$ in a ciprofloxacin-resistant strain of Typhimurium isolated after ciprofloxacin therapy. Recently, Piddock et al. [5], studying ciprofloxacin resistance in clinical isolates of Typhimurium obtained from two patients treated with ciprofloxacin, found no correlation between the lack of OMP porin $F$ and the decreased levels of quinolone accumulation. Multiple antibiotic resistance (Mar) phenotype has been implicated as the mechanism by which an organism might become resistant to quinolones and other antibiotics in E. coli [30]. The mar locus has also been found in Typhimurium [31]; however, mar mutants also show resistance to ampicillin, tetracycline and chloramphenicol. The strains analysed in this study were susceptible to the above mentioned antibiotics, suggesting that in these cases the mar locus is not involved in resistance to nalidixic acid.

The present study highlights the amino acid-sequence conservation of the quinolone resistance-determining region of GyrA protein of Typhimurium and E. coli. These results are in agreement with those of Reyna et al. [7], with spontaneous quinolone-resistant mutants of Typhimurium strain Su694, and of Brown et al. [8]. It has been shown that several amino-acid residues located between positions 67 and 106 are involved in the susceptibility of gram-negative and gram-positive species to quinolones [32]. However, the possible involvement of mutations in Ala-119, as the amino acid responsible for quinolone resistance in Typhimurium, has been suggested [33]. Among these residues Ser-83 in E. coli, or its equivalent in other species, seems to play a key role. Thus, the Ser- $83 \rightarrow$ Phe gyr A gene mutation appears to be commonly associated with clinical resistance to nalidixic acid in Typhimurium as shown in this study. However, Brown et al. [8] found this mutation in only one of the nine nalidixic acid-resistant isolates of Typhimurium, the other eight presenting an Asp-87 mutation to Gly or Asn; they also demonstrated a mutation in gyrA gene of serotype Typhi, i.e., Ser- 83 which changed to Phe [34]. The $\operatorname{parC}$ gene has also been implicated in the resistance to quinolones in $E$. coli $[9,10]$. In the present study, no changes in the region of parC homologous to the quinolone resistance-determining region of the gyrA gene were found in Typhimurium. These results clearly suggest that $\operatorname{par} \mathrm{C}$ mutations are not necessary to obtain a high level of resistance to nalidixic acid, although mutations in parC might be necessary to obtain a high level of resistance to fluoroquinolones.

In conclusion, on studying quinolone-resistant outbreak strains of Typhimurium, with a high level of resistance to nalidixic acid and a low level of 
resistance to fluoroquinolones, this study shows that a mutation in the gyrA gene is sufficient to impact high-level resistance to nalidixic acid.

This work was supported in part by grants PM93/1229 and FIS $95 / 0875$.

\section{References}

1. Asperilla MO, Smego RA, Scott LK. Quinolone antibiotics in the treatment of Salmonella infections. Rev Infect Dis 1990; 12: $873-889$

2. Barnass S, Franklin J, Tabaqchali S. The successful treatment of multiresistant non-enteric salmonellosis with seven day oral ciprofloxacin. J Antimicrob Chemother 1990; 25: 299-300.

3. Pichler HET, Diridl G, Stickler K, Wolf D. Clinical efficacy of ciprofloxacin compared with placebo in bacterial diarrhea. $\mathrm{Am}$ $J$ Med 1987; 82 Suppl 4A: 329-332.

4. Neill MA, Opal SM Heelan J et al. Failure of ciprofloxacin to eradicate convalescent fecal excretion after acute salmonellosis: experience during an outbreak in health care workers. Ann Intern Med 1991; 114: 195-199.

5. Piddock LJV, Griggs DJ, Hall MC, Jin YF. Ciprofloxacin resistance in clinical isolates of Salmonella typhimurium obtained from two patients. Antimicrob Agents Chemother 1993; 37: 662-666.

6. Heisig P. High-level fluoroquinolone resistance in a Salmonella typhimurium isolate due to alterations in both gyr $\mathrm{A}$ and gyr $\mathrm{B}$ genes. J Antimicrob Chemother 1993; 32: 367-377.

7. Reyna F, Huesca M, González V, Fuchs LY. Salmonella typhimurium gyrA mutations associated with fluoroquinolone resistance. Antimicrob Agents Chemother 1995; 39: 16211623.

8. Brown JC, Thomson CJ, Amyes SGB. Mutations of the gyrA gene of clinical isolates of Salmonella typhimurium and three other Salmonella species leading to decreased susceptibilities to 4-quinolone drugs. J Antimicrob Chemother 1996; 37: 351 356.

9. Khodursky AB, Zechiedrich EL, Cozzarelli NR. Topoisomerase IV is a target of quinolones in Escherichia coli. Proc Natl Acad Sci USA 1995; 92: 11801-11805.

10. Vila J, Ruiz J, Goñi P, Jimenez de Anta MT. Detection of mutations in parC gene of quinolone-resistant clinical isolates of Escherichia coli. Antimicrob Agents Chemother 1996; 40: 491-493.

11. Kado CI, Liu ST. Rapid procedure for detection and isolation of large and small plasmids. J Bacteriol 1981; 145: 13651373.

12. Marcos MA, Jimenez de Anta MT, Vila J. Correlation of six methods for typing nosocomial isolates of Acinetobacter baumannii. J Med Microbiol 1995; 42: 328-335.

13. National Committee for Clinical Laboratory Standards. Methods for dilution antimicrobial susceptibility tests for bacteria that grow aerobically, 2nd edn. Approved standard M7-A2. Villanova, PA, National Committee for Clinical Laboratory Standards. 1990.

14. National Committee for Clinical Laboratory Standards. Performance standards for antimicrobial disk susceptibility tests. M2A4, 4th edn. Villanova, PA, National Committee for Clinical Laboratory Standards. 1990.

15. Mortimer PGS, Piddock LJV. A comparison of methods used for measuring the accumulation of quinolones by Enterobacteriaceae, Pseudomonas aeruginosa and Staphylococcus aureus. J Antimicrob Chemother 1991; 28: 639-653.

16. Chart H, Trust TJ. Characterization of the surface antigens of the marine fish pathogens Vibrio anguillarum, and Vibrio ordalii. Can J Microbiol 1984; 30: 703-710.

17. Filip C, Fletcher G, Wulff JL, Earhart CF. Solubilization of the cytoplasmic membrane of Escherichia coli by the ionic detergent sodium-lauryl sarcosinate. $J$ Bacteriol 1973; 115: $717-722$.

18. Hitchcock PJ, Brown TM. Morphological heterogenecity among Salmonella lipopolysaccharide chemotypes in silverstained polyacrylamide gels. J Bacteriol 1983; 154: 269-277.

19. Tsai CM, Frasch CE. A sensitive silver stain for detecting lipopolysaccharides in polyacrylamide gels. Anal Biochem 1982; 119: 115-119.

20. Vila J, Ruiz J, Marco F et al. Association between double mutation in gyrA gene of ciprofloxacin-resistant clinical isolates of Escherichia coli and MICs. Antimicrob Agents Chemother 1994; 38: 2477-2479.

21. Swanberg SL, Wang JC. Cloning and sequencing of the Escherichia coli gyrA coding for the A subunit of DNA gyrase. J Mol Biol 1987; 197: 729-736.

22. Luttinger AL, Springer AL, Schmid MB. A cluster of genes that affects nucleoid segregation in Salmonella typhimurium. New Biol 1991; 3: 687-697.

23. Borrego JJ, Castro D, Jimenez-Notario $\mathrm{M}$ et al. Comparison of epidemiological markers of Salmonella strains isolated from different sources in Spain. J Clin Microbiol 1992; 30: 3058-3064.

24. Holmberg SD, Wachsmuth IK, Hickman-Brenner FW, Cohen ML. Comparison of plasmid profile analysis, phage typing, and antimicrobial susceptibility testing in characterizing Salmonella typhimurium isolates from outbreaks. J Clin Microbiol 1984; 19: $100-104$

25. Liu S-L, Hessel A, Sanderson KE. The $X b a \mathrm{I}-B \ln \mathrm{I}-\mathrm{Ceu} \mathrm{I}$ genomic cleavage map of Salmonella typhimurium LT2 determined by double digestion, end labelling, and pulsedfield electrophoresis. $J$ Bacteriol 1993; 175: 4104-4120.

26. Liu S-L, Sanderson KE. A physical map of the Salmonella typhimurium LT2 genome made by using $X b a \mathrm{I}$ analysis. $J$ Bacteriol 1992; 174: 1662-1672.

27. Hirai $\mathrm{K}$, Aoyama $\mathrm{H}$, Irikura $\mathrm{T}$, Iyobe $\mathrm{S}$, Mitsuhashi $\mathrm{S}$. Differences in susceptibility to quinolones of outer membrane mutants of Salmonella typhimurium and Escherichia coli. Antimicrob Agents Chemother 1986; 29: 535-538.

28. Lewin CS, Nandivada LS, Amyes SGB. Multiresistant salmonella and fluoroquinolones. $J$ Antimicrob Chemother 1991; 27: 147-149.

29. Howard AJ, Joseph TD, Bloodworth LLO, Frost JA, Chart H, Rowe B. The emergence of ciprofloxacin resistance in Salmonella typhimurium. J Antimicrob Chemother 1990; 26: 296-298.

30. Cohen SP, McMurry LM, Hooper DC, Wolfson JS, Levy SB. Cross-resistance to fluoroquinolones in multiple-antibioticresistant (Mar) Escherichia coli selected by tetracycline or chloramphenicol: decreased drug accumulation associated with membrane changes in addition to OmpF reduction. Antimicrob Agents Chemother 1989; 33: 1318-1325.

31. Cohen SP, Yan W, Levy SB. A multidrug resistance regulatory chromosomal locus is widespread among enteric bacteria. $J$ Infect Dis 1993; 168: 484-488.

32. Hooper DC, Wolfson FC. Mechanisms of bacterial resistance to quinolones. In: Hooper DC, Wolfson JC (eds) Quinolone antimicrobial agents, 2nd edn. Washington, DC. American Society for Microbiology. 1993: 97-119.

33. Griggs DJ, Gensberg K, Piddock LJ. Mutations in gyr A gene of quinolone-resistant Salmonella serotypes isolated from humans and animals. Antimicrob Agents Chemother 1996; 40: 1009-1013.

34. Brown JC, Shanahah PHA, Jesudason MV, Thomson CJ. Mutations responsible for reduced susceptibility to 4-quinolones in clinical isolates of multi-resistant Salmonella typhi in India. J Antimicrob Chemother 1996; 37: 891-900. 